\title{
Nonextensive statistics of Landsberg-Vedral entropy
}

\author{
A.S. Parvan ${ }^{1,2,3, *}$ \\ ${ }^{1}$ Joint Institute for Nuclear Research, Dubna, Russia \\ ${ }^{2}$ Horia Hulubei National Institute of Physics and Nuclear Engineering, Magurele, Romania \\ ${ }^{3}$ Institute of Applied Physics, Moldova Academy of Sciences, Chisinau, Republic of Moldova
}

\begin{abstract}
The general formalism for the nonextensive statistics based on the Landsberg-Vedral parametric entropy in the framework of the microcanonical, canonical and grand canonical ensembles was derived. The formulas for the first law of thermodynamics and the thermodynamic quantities in the terms of ensemble averages were obtained in a general form. It was found that under the transformation $q \rightarrow 2-q$ the probabilities of microstates of the nonextensive statistics based on the Landsberg-Vedral entropy with the standard expectation values formally resemble the corresponding probabilities of the Tsallis statistics with the generalized expectation values.
\end{abstract}

\section{Introduction}

Initially, the equilibrium statistical mechanics was implemented on the basis of the Boltzmann entropy, that established the connection between the macroscopic variable, entropy, and the number of microstates of a system [1,2] (see also [3]) and on the basis of the more general Gibbs formula, $S=-k_{B} \sum_{i} p_{i} \ln p_{i}$, that is the entropy or "uncertainty" of the probability distribution for the microstates of a system [4]. Lately, the Shannon expression [5] for the information entropy of the discrete probability distribution allowed E.T. Jaynes to give another formulation of the equilibrium statistical mechanics based on the maximum entropy principle or the second law of thermodynamics [6]. The Rényi alternative method of defining the information entropy $[7,8]$ opened a way for the introduction of new definitions of statistical entropies in the information theory (see, for instance, [3]). They had a significant impact on the development of the statistical models beyond the Boltzmann-Gibbs equilibrium statistical mechanics. The paper of C. Tsallis [9] established a new direction in science showing that on the basis of the Havrda-Charvát-Daroczy-Tsallis parametric entropy $[3,10,11]$ it is possible to construct the stationary statistical mechanics.

The Tsallis nonextensive statistics $[9,12]$ has received a wide recognition since it is confirmed by the experiment especially in high energy physics [13-27]. At the same time, the Boltzmann-Gibbs statistics faces difficulties in describing real phenomena, for example, in nuclear processes at high energies since equilibrium statistical mechanics is a simplified ideal limiting case of the behavior of reality [6]. The equilibrium theory neglects complex processes and phenomena which take place in real conditions. However, nonextensive stationary statistics such as the Tsallis statistics covers these complex phenomena and describes them parametrically due to the fact that a theory like that goes beyond the framework of

\footnotetext{
*e-mail: parvan@theor.jinr.ru,parvan@theory.nipne.ro
} 
the idealized Boltzmann-Gibbs statistics. The nonextensive parametrization may indicate the presence of complex processes in real phenomena such as, for example, nontrivial macromotions, particle transformation reactions, jets, internal currents in the system and etc. Nevertheless, the Tsallis statistics may have some indeterminacy in its formulation related to the generalized expectation values and the normalization condition for the probabilities of microstates $[12,28-30]$. The main aim of this paper is to introduce the nonextensive statistics based on the Landsberg-Vedral entropy [31] which may be formulated on the basis of the standard linear expectation values consistent with the normalization condition for the probabilities of microstates. The Landsberg-Vedral entropy is defined as [31]

$$
S=\frac{k_{B}}{1-q}\left(1-\frac{1}{\sum_{i} p_{i}^{q}}\right), \quad q>0,
$$

where $p_{i}$ is the probability of the $i$-th microscopic state of the system. The main important properties of the Landsberg-Vedral entropy that can be mentioned are: positivity $S \geq 0$, concavity in $\left\{p_{i}\right\}$, the Gibbs $\operatorname{limit}_{q \rightarrow 1} S=-k_{B} \sum_{i} p_{i} \ln p_{i}$, and the peculiar nonextensive additivity rule [31], $S(A+B)=S(A)+S(B)+\frac{q-1}{k_{B}} S(A) S(B)$, for two independent events $A$ and $B$.

\section{Microcanonical ensemble}

The thermodynamic potential of the microcanonical ensemble is the entropy. Let us consider the Landsberg-Vedral entropy (1) which was introduced in [31]. The probabilities of microstates are constrained by the additional function

$$
\varphi=\sum_{i} p_{i}-1=0
$$

and are obtained from the constrained local extrema of the thermodynamic potential (1) by the method of the Lagrange multipliers [32], $\Phi=S-\lambda \varphi$, and

$$
\frac{\partial \Phi}{\partial p_{i}}=0
$$

where $\lambda$ is an arbitrary real constant. Substituting Eqs. (1) and (2) into $\Phi$ and using Eqs. (3) and (2), we obtain $p_{i}=1 / W$ and $W=\sum_{i} \delta_{E, E_{i}} \delta_{V, V_{i}} \delta_{V, V_{i}}$, where $E_{i}, V_{i}$ and $N_{i}$ are energy, volume and number of particles, respectively, in the $i$-th microscopic state of the system. Then, the thermodynamic potential (1) can be rewritten as (cf. refs. [1, 2, 33])

$$
S=\frac{W^{q-1}-1}{q-1}=z\left(W^{1 / z}-1\right), \quad z \equiv \frac{1}{q-1} .
$$

Note that here and throughout the paper we use the system of natural units $\hbar=c=k_{B}=1$.

Let us suppose that $z$ is a variable of state of the system. Then, we obtain $d S=\frac{d z}{z}(S-$ $\left.W^{1 / z} \ln W\right)+W^{1 / z} d \ln W$. The statistical weight $W=W(E, V, N)$. Therefore, we have

$$
\begin{aligned}
T d S & =d E+p d V+X d z-\mu d N \\
\frac{1}{T} & =W^{1 / z} \frac{\partial \ln W}{\partial E}=\left(\frac{\partial S}{\partial E}\right)_{V z N}, \quad \frac{p}{T}=W^{1 / z} \frac{\partial \ln W}{\partial V}=\left(\frac{\partial S}{\partial V}\right)_{E z N} \\
-\frac{\mu}{T} & =W^{1 / z} \frac{\partial \ln W}{\partial N}=\left(\frac{\partial S}{\partial N}\right)_{E V z}, \quad \frac{X}{T}=W^{1 / z}-1-W^{1 / z} \ln W^{1 / z}=\left(\frac{\partial S}{\partial z}\right)_{E V N} .
\end{aligned}
$$


The first partial derivatives of the thermodynamic potential $S$ with respect to the variables of state in Eqs. (6)-(7) can also be verified from Eq. (4). Equation (5) represents the first law of thermodynamics. In the limit $q \rightarrow 1$ we obtain all the relations of the Boltzmann-Gibbs statistics.

\section{Canonical ensemble}

The thermodynamic potential of the canonical ensemble, the free energy $F$, is obtained from the fundamental thermodynamic potential, the energy $E$, by the Legendre transform. Using Eq. (1), we have

$$
F=E-T S=\sum_{i} p_{i} E_{i}-\frac{T}{1-q}\left(1-\frac{1}{\sum_{i} p_{i}^{q}}\right),
$$

where $E=\sum_{i} p_{i} E_{i}$. Substituting Eqs. (8) and (2) into the Lagrange function of the canonical ensemble [34], $\Phi=F-\lambda \varphi$, and using Eqs. (3) and (2), we obtain

$$
p_{i}=\frac{1}{Z_{c}}\left[1+(q-1) \frac{\Lambda-E_{i}}{T}\right]^{\frac{1}{q-1}}, \quad Z_{c}=\sum_{i}\left[1+(q-1) \frac{\Lambda-E_{i}}{T}\right]^{\frac{1}{q-1}}, \quad Z_{c}^{q-1}=\frac{q}{\chi^{2}},
$$

where $\chi \equiv \sum_{i} p_{i}^{q}, \lambda$ is an arbitrary real constant and $\Lambda=\lambda-T /(q-1)$. Substituting $p_{i}$ into $\chi$ and using Eq. (9), we find an equation for $\Lambda$ as

$$
\sum_{i}\left[1+(q-1) \frac{\Lambda-E_{i}}{T}\right]^{\frac{1}{q-1}}=\left\{q^{-1 / 2} \sum_{i}\left[1+(q-1) \frac{\Lambda-E_{i}}{T}\right]^{\frac{q}{q-1}}\right\}^{\frac{2}{q+1}} .
$$

Substituting $p_{i}$ into Eqs. (1) and (8), and using Eq. (2), we have

$$
\begin{aligned}
S & =\frac{1}{1+(q-1) \frac{\Lambda-E}{T}}\left[\frac{Z_{c}^{q-1}-1}{q-1}-\frac{\Lambda-E}{T}\right] \\
F & =\Lambda-\frac{T}{1+(q-1) \frac{\Lambda-E}{T}}\left[\frac{Z_{c}^{q-1}-1}{q-1}+(q-1)\left(\frac{\Lambda-E}{T}\right)^{2}\right] .
\end{aligned}
$$

In the canonical ensemble the entropy $S=S(T, V, z, N)$. Thus, using Eq. (1) and the definition of $z$ given in Eq. (4), we obtain

$$
d S=\frac{d z}{z}\left(S+\frac{1}{\chi^{2}} \sum_{i} p_{i}^{1+\frac{1}{z}} \ln p_{i}\right)-\frac{z\left(1+\frac{1}{z}\right)}{\chi^{2}} \sum_{i} p_{i}^{\frac{1}{z}} d p_{i}
$$

Substituting $p_{i}$ into Eq. (13) and using $\sum_{i} d p_{i}=0$ and Eq. (9), we can write

$$
d S=\frac{d z}{z}\left(S+\frac{1}{\chi^{2}} \sum_{i} p_{i}^{1+\frac{1}{z}} \ln p_{i}\right)+\frac{1}{T} \sum_{i} E_{i} d p_{i}
$$

The differential of the energy $E$ can be written as

$$
d E=\sum_{i} p_{i} d E_{i}+\sum_{i} E_{i} d p_{i}, \quad d E_{i}=\frac{\partial E_{i}}{\partial V} d V+\frac{\partial E_{i}}{\partial N} d N
$$


where $\partial E_{i} / \partial T=\partial E_{i} / \partial z=0$. Then, substituting Eq. (15) into Eq. (14), we obtain the first law of thermodynamics (cf. [35] for the Tsallis statistics)

$$
\begin{aligned}
T d S & =d E+p d V+X d z-\mu d N \\
p & =-\sum_{i} p_{i} \frac{\partial E_{i}}{\partial V}, \quad \mu=\sum_{i} p_{i} \frac{\partial E_{i}}{\partial N}, \quad X=\frac{T}{z}\left(S+\frac{1}{\chi^{2}} \sum_{i} p_{i}^{1+\frac{1}{z}} \ln p_{i}\right) .
\end{aligned}
$$

Then, from Eq. (16) and the Legendre transform (8) the differential thermodynamic relation for the thermodynamic potential $F$ is $d F=-S d T-p d V-X d z+\mu d N$.

Taking the first derivative of the thermodynamic potential (8) with respect to temperature $T$, volume $V$, number of particles $N$ and variable $z$, and using Eq. (9), and the relations $\partial E_{i} / \partial T=0, \sum_{i} \partial p_{i} / \partial T=0, \sum_{i} \partial p_{i} / \partial V=0, \sum_{i} \partial p_{i} / \partial N=0, \partial E_{i} / \partial z=0, \sum_{i} \partial p_{i} / \partial z=0$, we obtain the entropy $S$, the pressure $p$, the chemical potential $\mu$ and the conjugate force $X$, respectively, as

$$
\begin{aligned}
S & =-\left(\frac{\partial F}{\partial T}\right)_{V z N}=\frac{1}{1-q}\left(1-\frac{1}{\sum_{i} p_{i}^{q}}\right), \quad p=-\left(\frac{\partial F}{\partial V}\right)_{T z N}=-\sum_{i} p_{i} \frac{\partial E_{i}}{\partial V}, \\
\mu & =\left(\frac{\partial F}{\partial N}\right)_{T V z}=\sum_{i} p_{i} \frac{\partial E_{i}}{\partial N}, \quad X=-\left(\frac{\partial F}{\partial z}\right)_{T V N}=\frac{T}{z}\left(S+\frac{1}{\chi^{2}} \sum_{i} p_{i}^{1+\frac{1}{z}} \ln p_{i}\right) .
\end{aligned}
$$

Applying the Legendre back-transformation to the function $F$ and using Eqs. (8) and (18), we obtain the mean energy of the system as

$$
E=-T^{2}\left(\frac{\partial}{\partial T} \frac{F}{T}\right)_{V z N}=\sum_{i} p_{i} E_{i}
$$

Let us rewrite the probabilities of microstates (9) in another representation. Substituting Eq. (9) into the function $\chi$ and using the relation for $Z_{c}$ and $\chi$, we obtain $1+(q-1) \frac{\Lambda}{T}=$ $\frac{q}{\chi}+(q-1) \frac{E}{T}$. Substituting this equation into Eq. (9), we have

$$
p_{i}=\frac{1}{Z_{q}}\left[1+(q-1) \frac{E-E_{i}}{c T}\right]^{\frac{1}{q-1}}, \quad Z_{q}=\sum_{i}\left[1+(q-1) \frac{E-E_{i}}{c T}\right]^{\frac{1}{q-1}}, \quad c=q Z_{q}^{q-1},
$$

where $\chi=q / c$. It can be observed that under the transformation $q \rightarrow 2-q$ the probability of microstates (21) formally recovers the probability of microstates of the Tsallis statistics with escort probabilities $[12,36]$. Note that in the Gibbs limit $q \rightarrow 1$ we obtain all the relations of the Boltzmann-Gibbs statistics in the canonical ensemble and the parameter $X$ vanishes.

\section{Grand canonical ensemble}

The thermodynamic potential of the grand canonical ensemble is related to the fundamental thermodynamic potential, energy $E$, by the Legendre transform. Using Eq. (1), we have

$$
\Omega=E-T S-\mu N=\sum_{i} p_{i}\left(E_{i}-\mu N_{i}\right)-\frac{T}{1-q}\left(1-\frac{1}{\sum_{i} p_{i}^{q}}\right),
$$

where $E=\sum_{i} p_{i} E_{i}$ and $N=\sum_{i} p_{i} N_{i}$. Substituting Eqs. (22) and (2) into the Lagrange function of the grand canonical ensemble [30], $\Phi=\Omega-\lambda \varphi$, and using Eqs. (3) and (2), we obtain

$$
p_{i}=\frac{1}{Z_{\Omega}}\left[1+(q-1) \frac{\Lambda-E_{i}+\mu N_{i}}{T}\right]^{\frac{1}{q-1}}, \quad Z_{\Omega}=\sum_{i}\left[1+(q-1) \frac{\Lambda-E_{i}+\mu N_{i}}{T}\right]^{\frac{1}{q-1}},
$$


where $Z_{\Omega}^{q-1}=q / \chi^{2}, \lambda$ is an arbitrary real constant and $\chi$ and $\Lambda$ are defined below Eq. (9). Substituting $p_{i}$ into $\chi$ and using $Z_{\Omega}$ and the relation between $Z_{\Omega}$ and $\chi$, we found an equation for $\Lambda$ as

$$
\sum_{i}\left[1+(q-1) \frac{\Lambda-E_{i}+\mu N_{i}}{T}\right]^{\frac{1}{q-1}}=\left\{q^{-1 / 2} \sum_{i}\left[1+(q-1) \frac{\Lambda-E_{i}+\mu N_{i}}{T}\right]^{\frac{q}{q-1}}\right\}^{\frac{2}{q+1}} .
$$

Substituting Eq. (23) into Eqs. (1) and (22), and using Eq. (2), we have

$$
\begin{aligned}
S & =\frac{1}{1+(q-1) \frac{\Lambda-E+\mu N}{T}}\left[\frac{Z_{\Omega}^{q-1}-1}{q-1}-\frac{\Lambda-E+\mu N}{T}\right], \\
\Omega & =\Lambda-\frac{T}{1+(q-1) \frac{\Lambda-E+\mu N}{T}}\left[\frac{Z_{\Omega}^{q-1}-1}{q-1}+(q-1)\left(\frac{\Lambda-E+\mu N}{T}\right)^{2}\right] .
\end{aligned}
$$

In the grand canonical ensemble the entropy $S=S(T, V, z, \mu)$. Thus, using Eq. (1) and the definition of $z$ given in Eq. (4), we obtain exactly Eq. (13). Substituting Eq. (23) into Eq. (13) and using $\sum_{i} d p_{i}=0$ and the relation between $Z_{\Omega}$ and $\chi$, we obtain

$$
d S=\frac{X}{T} d z+\frac{1}{T} \sum_{i}\left(E_{i}-\mu N_{i}\right) d p_{i}
$$

where $X$ is the same as in Eq. (17). The differential of the mean energy $E$ and the mean number of particles $N$ can be written as

$$
d E-\mu d N=\sum_{i} p_{i}\left(d E_{i}-\mu d N_{i}\right)+\sum_{i}\left(E_{i}-\mu N_{i}\right) d p_{i}
$$

where $d E_{i}-\mu d N_{i}=\left(\frac{\partial E_{i}}{\partial V}-\mu \frac{\partial N_{i}}{\partial V}\right) d V, \partial E_{i} / \partial T=\partial E_{i} / \partial z=\partial E_{i} / \partial \mu=0$ and $\partial N_{i} / \partial T=$ $\partial N_{i} / \partial z=\partial N_{i} / \partial \mu=0$. Then, substituting Eqs. (28) into Eq. (27), we obtain the first law of thermodynamics

$$
T d S=d E+p d V+X d z-\mu d N, \quad p=-\sum_{i} p_{i}\left(\frac{\partial E_{i}}{\partial V}-\mu \frac{\partial N_{i}}{\partial V}\right) .
$$

Then, from Eq. (29) and the Legendre transform (22) we have $d \Omega=-S d T-p d V-X d z-N d \mu$.

Taking the first derivative of the thermodynamic potential (22) with respect to temperature $T$, volume $V$, chemical potential $\mu$ and variable $z$, and using Eq. (23) and the relation between $Z_{\Omega}$ and $\chi$, and $\partial E_{i} / \partial T=\partial N_{i} / \partial T=0, \sum_{i} \partial p_{i} / \partial T=0$, we obtain the entropy $S$, pressure $p$, mean number of particles $N$ and conjugate force $X$, respectively, as

$$
\begin{aligned}
& S=-\left(\frac{\partial \Omega}{\partial T}\right)_{V z \mu}=\frac{1}{1-q}\left(1-\frac{1}{\sum_{i} p_{i}^{q}}\right), p=-\left(\frac{\partial \Omega}{\partial V}\right)_{T z \mu}=-\sum_{i} p_{i}\left(\frac{\partial E_{i}}{\partial V}-\mu \frac{\partial N_{i}}{\partial V}\right) \\
& N=-\left(\frac{\partial \Omega}{\partial \mu}\right)_{T V z}=\sum_{i} p_{i} N_{i}, \quad X=-\left(\frac{\partial \Omega}{\partial z}\right)_{T V \mu}=\frac{T}{z}\left(S+\frac{1}{\chi^{2}} \sum_{i} p_{i}^{1+\frac{1}{z}} \ln p_{i}\right) .
\end{aligned}
$$

Applying the Legendre back-transformation to the function $\Omega$ and using Eqs. (22) and (30), we obtain the mean energy of the system as

$$
E=-T^{2}\left(\frac{\partial}{\partial T} \frac{\Omega}{T}\right)_{V z \mu}+\mu N=\sum_{i} p_{i} E_{i}
$$

In the Gibbs limit $q \rightarrow 1$ we obtain all the relations of the Boltzmann-Gibbs statistics in the grand canonical ensemble and the parameter $X$ vanishes, $X=0$. 


\section{Conclusions}

In conclusion, we have introduced new nonextensive statistics based on the Landsberg-Vedral entropy. In its formulation we have used the standard linear expectation values constrained with the standard normalization condition for the probabilities of microscopic states of the system. The three ensembles were considered: microcanonical, canonical and grand canonical. The probabilities of microstates were obtained from the principle of maximum entropy using the method of the Lagrange multipliers. In each of these three ensembles we have derived exactly the first law of thermodynamics. We have also obtained the exact relations between the thermodynamic definitions of the thermodynamic quantities and their ensemble averages. The ensemble averages for the pressure, the chemical potential, the mean number of particles and the mean energy in both canonical and grand canonical ensembles are the same as those of the Boltzmann-Gibbs statistics with the exception of the form of the probability of microstates. In this formalism the Legendre transform is preserved. We have found that under the transformation $q \rightarrow 2-q$ the probabilities of microstates for the nonextensive statistics based on the Landsberg-Vedral entropy formally recover the probabilities of microstates of the Tsallis statistics with escort probabilities. However, the nonextensive statistics based on the Landsberg-Vedral entropy does not require introduction of the complicated escort probabilities and generalized expectation values which lie in the definition of the Tsallis statistics with escort probabilities.

Acknowledgments. This work was supported in part by the joint research project of JINR and IFIN-HH.

\section{References}

[1] L. Boltzmann, Wiener Ber. 75, 67 (1877)

[2] L. Boltzmann, Wiener Ber. 76, 373 (1877)

[3] A. Wehrl, Rev. Mod. Phys. 50, 221 (1978)

[4] E.T. Jaynes, Am. J. Phys. 33, 391 (1965)

[5] C.E. Shannon, Bell System Tech. J. 27, 379 (1948)

[6] E.T. Jaynes, Phys. Rev. 106, 620 (1957)

[7] A. Rényi, in Proc. Fourth Berkeley Symposium on Mathematical Statistics and Probability, I, 547 (1961)

[8] A. Rényi, Probability Theory (North-Holland, Amsterdam, 1970)

[9] C. Tsallis, J. Stat. Phys. 52, 479 (1988)

[10] J. Havrda, F. Charvát, Kybernetica 3, 30 (1967)

[11] Z. Daroczy, Inf. Control 16, 36 (1970)

[12] C. Tsallis, R.S. Mendes, A.R. Plastino, Physica A 261, 534 (1998)

[13] K. Aamodt et al. (ALICE Collaboration), Eur. Phys. J. C 71, 1655 (2011)

[14] G. Aad et al. (ATLAS Collaboration), New J. Phys. 13, 053033 (2011)

[15] V. Khachatryan et al. (CMS Collaboration), Phys. Rev. Lett. 105, 022002 (2010)

[16] M. Rybczyński, Z. Włodarczyk, Eur. Phys. J. C 74, 2785 (2014)

[17] J. Cleymans, G.I. Lykasov, A.S. Parvan, A.S. Sorin, O.V. Teryaev, D. Worku, Phys. Lett. B 723, 351 (2013)

[18] M.D. Azmi, J. Cleymans, J. Phys. G: Nucl. Part. Phys. 41, 065001 (2014)

[19] J. Cleymans, D. Worku, J. Phys. G: Nucl. Part. Phys. 39, 025006 (2012)

[20] J. Cleymans, D. Worku, Eur. Phys. J. A 48, 160 (2012)

[21] L. Marques, E. Andrade-II, A. Deppman, Phys. Rev. D 87, 114022 (2013) 
[22] B.-C. Li, Y.-Z. Wang, F.-H. Liu, X.-J. Wen and Y.-E. Dong, Phys. Rev. D 89, 054014 (2014)

[23] A.S. Parvan, PoS Baldin ISHEPP XXII, 077 (2015)

[24] A.S. Parvan, Eur. Phys. J. A 52, 355 (2016)

[25] A.S. Parvan, O.V. Teryaev, J. Cleymans, Eur. Phys. J. A 53, 102 (2017)

[26] M. Biyajima, T. Mizoguchi, N. Nakajima, N. Suzuki, G. Wilk, Eur. Phys. J. C 48, 597 (2006)

[27] L. Marques, J. Cleymans, A. Deppman, Phys. Rev. D 91, 054025 (2015)

[28] A. Plastino, A.R. Plastino, Phys. Lett. A 226, 257 (1997)

[29] S. Abe, Phys. Lett. A 275, 250 (2000)

[30] A.S. Parvan, Eur. Phys. J. A 51, 108 (2015)

[31] P.T. Landsberg, V. Vedral, Phys. Lett. A 247, 211 (1998)

[32] M.L. Krasnov, G.I. Makarenko, A.I. Kiseliov, Calculus of Variations: Problems and Exercises with detailed solutions (URSS Publisher, Moscow 2002)

[33] A.S. Parvan, Phys. Lett. A 350, 331 (2006)

[34] A.S. Parvan, in Recent Advances in Thermo and Fluid Dynamics, ed. by Mofid GorjiBandpy (InTech, Rijeka, 2015) 303

[35] A.S. Parvan, Phys. Lett. A 360, 26 (2006)

[36] S. Abe, Physica A 269, 403 (1999) 